\title{
Combination of Arginine Depletion and Chemotherapy in Thoracic Malignancies
}

\author{
James Chung-Man Ho and Sze-Kwan Lam, The University of Hong Kong, Hong Kong, Special Administrative Region, People's \\ Republic of China
}

See accompanying article on page 1778

Thoracic malignancies, especially lung cancer and malignant pleural mesothelioma (MPM), account for a huge disease burden with devastating mortality worldwide. The unprecedented advances in the development of anticancer therapies in the past few years reflect the importance of precision medicine. For advanced non-small-cell lung cancer (NSCLC), the current standard of care is to determine the presence of actionable targets, such as epidermal growth factor receptor mutations and anaplastic lymphoma kinase rearrangement, that allow logical use of specific targeted therapies. ${ }^{1-4}$ Recent data also support the upfront use of immuno-oncologic agents (pembrolizumab, an anti-programmed death 1 or PD1 antibody) among the subgroup of patients with advanced NSCLC that strongly expresses PD-L1. ${ }^{5}$ In contrast, the current standard therapeutic options for advanced MPM are limited, with pemetrexed platinum doublet as the standard of care, whereas emerging data support the potential role of bevacizumab ${ }^{6}$ and immuno-oncologic agents ${ }^{7}$ in recent years. An urgent need still exists for novel and personalized cancer therapeutics in both advanced lung cancer and MPM.

In the article that accompanies this editorial, Beddowes et $\mathrm{al}^{8}$ report a phase I trial of pegylated arginine deiminase (ADI-PEG $20)$, cisplatin, and pemetrexed in nine patients with advanced NSCLC or MPM who are deficient in argininosuccinate synthetase 1 (ASS1). Because ASS1 is the key enzyme involved in the urea cycle for biosynthesis of arginine, tumors deficient in ASS1 are predictably susceptible to therapeutic arginine depletion (ie, arginine auxotrophic) with agents like ADI-PEG 20. Apart from determining the recommended dose of ADI-PEG 20 for future study, this small phase I study also provides a glimpse of the clinical activity of this combination, especially in MPM. The approach in selecting tumors on the basis of ASS1 expression by immunohistochemistry (IHC) is biologically sound. However, because IHC expression of ASS1 is a continuum rather than a dichotomized biomarker, the exact cutoff values need to be clinically determined. Beddowes et $\mathrm{al}^{8}$ defined ASS 1 deficiency by 0 or $1+$ IHC staining in $>50 \%$ tumor cells, which is based on preclinical studies. ${ }^{9,10}$ The same definition of ASS1 deficiency has also been adopted in the Arginine Deiminase and Mesothelioma (ADAM) study, which reported favorable progression-free survival with ADI-PEG 20 monotherapy compared with best supportive care in ASS1deficient MPM. ${ }^{11}$ Nonetheless, the appropriate and clinically relevant cutoff that defines ASS1 deficiency requires a robust clinical investigation that preferably involves separate training and validation cohorts, which is lacking at the moment. The biologic response to arginine depletion also may vary between tumors, and whether the same cutoff can be used in different tumor types remains to be determined.

The main limitation that precludes sustained response to ADI-PEG 20 is the development of a neutralizing antibody. In the ADAM study with monotherapy of ADI-PEG 20 in MPM, approximately two thirds of patients developed an anti-ADI-PEG 20 antibody, which resulted in a rebound increase in plasma arginine level by the third month of treatment. ${ }^{11}$ Similarly, a previous phase I study of the combination of ADI-PEG 20 and docetaxel in various advanced solid tumors also detected autoantibodies to ADI-PEG 20 as early as 6 weeks after treatment, which resulted in a gradual return to baseline levels of plasma arginine. ${ }^{12}$ On the other hand, no neutralizing antibody production against ADI-PEG 20 was observed in early clinical trials in melanoma ${ }^{13}$ and hepatocellular carcinoma. ${ }^{14}$ Of note, Beddowes et $\mathrm{al}^{8}$ show that the combination of ADI-PEG 20, cisplatin, and pemetrexed can rapidly deplete plasma arginine levels and sustain these levels throughout the 18 weeks of treatment, although no data during ADI-PEG 20 monotherapy beyond the combination phase are reported. This finding is corroborated by the detection of a neutralizing antibody against ADI-PEG 20 by 8 to 10 weeks at low titers until 18 weeks of treatment. The exact explanation for the low level of antidrug antibody with this combination, in contrast to monotherapy with ADI-PEG 20 in the ADAM study, ${ }^{11}$ needs to be further elucidated but could be related to the use of corticosteroid premedication, chemotherapy-specific effects, or simply chance as a result of the small sample size. ${ }^{8}$ Future development of arginine depletion therapy with ADI-PEG 20 will hinge on the effective ways to prevent the production of neutralizing antidrug antibody.

Although one should avoid overemphasis of the clinical efficacy observed in a small-scale phase I study, Beddowes et $\mathrm{al}^{8}$ report a notable objective response rate of $78 \%$ with the combination therapy, which is unusual for biphasic and sarcomatoid MPM, and these encouraging results warrant confirmation in future large-scale randomized controlled trials. Compared with data from the ADAM study, the best response attained with ADIPEG 20 monotherapy at 4 months was only disease stabilization despite a predominance of nonsarcomatoid histology of MPM, ${ }^{11}$ which could be partly due to the rapid emergence of antidrug 
antibody that led to unsustained depletion of plasma arginine in the majority of subjects. Because continuation maintenance therapy with pemetrexed is an established standard of care in advanced nonsquamous NSCLC, future clinical trials also should explore the role of a maintenance doublet with ADI-PEG 20 and pemetrexed for patients who respond to triplet induction therapy.

In the ADAM study, some concerns exist about the safety of ADI-PEG 20 treatment, which was associated with grade 3 anaphylaxis in four patients (9\%) and with serum sickness in two. ${ }^{11}$ However, in the study by Beddowes et $\mathrm{al}^{8}$ the combination of ADIPEG 20, cisplatin, and pemetrexed was well tolerated. In the only patient who experienced an infusion-related adverse effect, the hypersensitivity reaction was ascribed to cisplatin rather than to ADIPEG 20. If this reaction is confirmed in future large-scale clinical trials, corticosteroid premedication similar to the usual premedication for pemetrexed should be considered for ADI-PEG 20 therapy.

Following in the footsteps of L-asparaginase in the treatment of acute lymphoblastic leukemia, arginine depletion therapy has now emerged as a novel therapeutic for biomarker-selected thoracic malignancies. Future development of ADI-PEG 20 as an arginine depletor with or without systemic chemotherapy will add to the growing armamentarium in the fight against advanced lung cancer and MPM.

\section{AUTHORS' DISCLOSURES OF POTENTIAL CONFLICTS OF INTEREST}

Disclosures provided by the authors are available with this article at jco.org.

\section{AUTHOR CONTRIBUTIONS}

Manuscript writing: All authors

Final approval of manuscript: All authors

\section{REFERENCES}

1. Mok TS, Wu YL, Thongprasert $S$, et al: Gefitinib or carboplatin-paclitaxel in pulmonary adenocarcinoma. N Engl J Med 361:947-957, 2009

2. Zhou C, Wu YL, Chen G, et al: Erlotinib versus chemotherapy as first-line treatment for patients with advanced EGFR mutation-positive non-small-cell lung cancer (OPTIMAL, CTONG-0802): A multicentre, open-label, randomised, phase 3 study. Lancet Oncol 12:735-742, 2011

3. Yang JC, Wu YL, Schuler $M$, et al: Afatinib versus cisplatin-based chemotherapy for EGFR mutation-positive lung adenocarcinoma (LUX-Lung 3 and LUX-Lung 6): Analysis of overall survival data from two randomised, phase 3 trials. Lancet Oncol 16:141-151, 2015

4. Solomon BJ, Mok T, Kim DW, et al: First-line crizotinib versus chemotherapy in ALK-positive lung cancer. N Engl J Med 371:2167-2177, 2014

5. Reck M, Rodríguez-Abreu D, Robinson AG, et al: Pembrolizumab versus chemotherapy for PD-L1-positive non-small-cell lung cancer. N Engl J Med 375: 1823-1833, 2016

6. Zalcman G, Mazieres J, Margery J, et al: Bevacizumab for newly diagnosed pleural mesothelioma in the Mesothelioma Avastin Cisplatin Pemetrexed Study (MAPS): A randomised, controlled, open-label, phase 3 trial. Lancet 387:1405-1414, 2016

7. Ceresoli GL, Bonomi M, Sauta MG: Immune checkpoint inhibitors in malignant pleural mesothelioma: Promises and challenges. Expert Rev Anticancer Ther $16: 673-675,2016$

8. Beddowes E, Spicer J, Chan PY, et al: Phase 1 dose-escalation study of pegylated arginine deiminase, cisplatin, and pemetrexed in patients with argininosuccinate synthetase 1-deficient thoracic cancers. J Clin Oncol 35:1778-1785, 2017

9. Szlosarek PW, Klabatsa A, Pallaska A, et al: In vivo loss of expression of argininosuccinate synthetase in malignant pleural mesothelioma is a biomarker for susceptibility to arginine depletion. Clin Cancer Res 12:7126-7131, 2006

10. Delage $B$, Fennell DA, Nicholson $L$, et al: Arginine deprivation and argininosuccinate synthetase expression in the treatment of cancer. Int J Cancer 126: 2762-2772, 2010

11. Szlosarek PW, Steele JP, Nolan L, et al: Arginine deprivation with pegylated arginine deiminase in patients with argininosuccinate synthetase 1-deficient malignant pleural mesothelioma: A randomized clinical trial. JAMA Oncol 3: 58-66, 2017

12. Tomlinson BK, Thomson JA, Bomalaski JS, et al: Phase I trial of arginine deprivation therapy with ADI-PEG 20 plus docetaxel in patients with advanced malignant solid tumors. Clin Cancer Res 21:2480-2486, 2015

13. Ascierto PA, Scala $S$, Castello G, et al: Pegylated arginine deiminase treatment of patients with metastatic melanoma: Results from phase I and II studies. J Clin Oncol 23:7660-7668, 2005

14. Izzo F, Marra $P$, Beneduce $G$, et al: Pegylated arginine deiminase treatment of patients with unresectable hepatocellular carcinoma: Results from phase I/II studies. J Clin Oncol 22:1815-1822, 2004

DOI: https://doi.org/10.1200/JC0.2017.72.7305; published at jco.org on April 14, 2017. 
AUTHORS' DISCLOSURES OF POTENTIAL CONFLICTS OF INTEREST

Combination of Arginine Depletion and Chemotherapy in Thoracic Malignancies

The following represents disclosure information provided by authors of this manuscript. All relationships are considered compensated. Relationships are self-held unless noted. I = Immediate Family Member, Inst = My Institution. Relationships may not relate to the subject matter of this manuscript. For more information about ASCO's conflict of interest policy, please refer to www.asco.org/rwc or ascopubs.org/jco/site/ifc.

James Chung-Man Ho

Honoraria: AstraZeneca, Roche, Pfizer, Novartis, Boehringer Ingelheim, Eli Lilly, MSD

Consulting or Advisory Role: AstraZeneca, Roche, Pfizer, Boehringer Ingelheim, Novartis, MSD

Speakers' Bureau: AstraZeneca, Novartis, Boehringer Ingelheim, MSD, Eli Lilly

Research Funding: AstraZeneca (Inst), Roche (Inst), Boehringer

Ingelheim (Inst), Novartis (Inst), MSD (Inst), Eli Lilly (Inst), Pfizer (Inst)

\section{Sze-Kwan Lam}

No relationship to disclose 